(c) American Dairy Science Association, 2004.

\title{
Genetic Variation and Heritability of the Antibody Response to Mycobacterium avium subspecies paratuberculosis in Danish Holstein Cows
}

\author{
H. Mortensen, ${ }^{1}$ S. S. Nielsen, ${ }^{1}$ and P. Berg ${ }^{2}$ \\ ${ }^{1}$ Department of Animal Science and Animal Health, \\ The Royal Veterinary and Agricultural University, \\ 1870 Frederiksberg C, Denmark \\ ${ }^{2}$ Danish Institute of Agricultural Science, \\ Department of Animal Breeding and Genetics, \\ 8830 Tjele, Denmark
}

\begin{abstract}
The purpose of this study was to estimate the genetic variation and the heritability of the ability to establish an immune response by producing antibodies to $\mathrm{Myco}$ bacterium avium subsp. paratuberculosis. Antibody levels were determined using an ELISA and measuring optical density (OD) values from milk samples of 11,535 cows from 99 herds. The pedigree of the 11,535 cows and information about days in milk, parity, milk yield, and others were obtained from the Danish Cattle database. The statistical analyses were made using a bivariate mixed animal model. The bivariate model with daily milk yield and OD as dependent variables showed a significant heritability of the ability to produce $\mathrm{Myco}$ bacterium avium subsp. paratuberculosis antibodies of 0.102 (genetic variance $=0.054$ ) and a nonsignificant genetic correlation of -0.037 between daily milk yield and OD. When a sire model was used, the estimated heritability was 0.091 . To evaluate whether intrauterine infection could be reflected in the OD, variation among 2715 dam-daughter or maternal sister groups was also estimated. Variation in this data was nonsignificant, possibly because only very few clinical cases or end-stage cows, i.e., heavy bacterial shedders may have been represented among the cows sampled. It does not appear that intrauterine transmission is of major importance in the transmission of paratuberculosis.
\end{abstract}

(Key words: heritability, paratuberculosis, ELISA, cattle)

Abbreviation key: $\mathbf{C M I}$ = cell-mediated immune response, $\mathbf{H I}=$ humoral immune response, $\mathbf{M a a}=$ Mycobacterium avium subsp. avium, Map = Mycobacterium avium subsp. paratuberculosis, NRAMP1 = Natural

Received September 2, 2003.

Accepted January 20, 2004.

Corresponding author: S. S. Nielsen; e-mail: ssn@kvl.dk. resistance associated macrophage protein $1, \mathbf{O D}=$ optical density.

\section{INTRODUCTION}

Paratuberculosis in ruminants, caused by Mycobacterium avium subsp. paratuberculosis (Map), is pathologically seen as chronic enteritis mainly in the small intestine. In cattle, the primary symptom is a profound malabsorption diarrhea with reduced of milk production and BW. The infection route is mainly oral, but evidence of intrauterine transmission has also been reported (Seitz et al., 1989; Sweeney et al., 1992). Calves are most susceptible to infection at 0 to 4 mo of age (Taylor, 1953), and the incubation period may vary from 2 to $10 \mathrm{yr}$ (Whitlock and Buergelt, 1996).

Mycobacterium avium subsp. paratuberculosis is an intracellular bacterium that survives inside the macrophages, resulting in a cell-mediated type immune response (CMI). During CMI, only small levels of humoral antibodies are produced. While CMI dominates, paratuberculosis is thought to be kept under control (Chiodini, 1996; Koets et al., 2001). When the humoral immune response (HI) for unknown reasons takes over, a concomitant increase in bacterial proliferation may occur (Chiodini, 1996). Some are of the opinion that HI is the dominant immune response during clinical disease (Stabel, 2000). When HI takes over, IgG1 antibodies will especially be produced (Koets et al., 2001).

The economic losses attributed to paratuberculosis are due to decreased milk production, reduced value of slaughter animals, and premature death in some cases. The economic loss has been estimated to US $\$ 50$ per cow in herds positive with Map (Chi et al., 2002). Others have estimated a loss of $\$ 100$ per cow by comparison of infected and noninfected herds (Ott et al., 1999). The herd prevalence of paratuberculosis varies between countries ranging from 0 to $71 \%$ of the herds being infected (reviewed in Kennedy and Benedictus, 2001). 
Table 1. The distribution of cows and samples collected from 99 Danish dairy herds in 3 sampling rounds. Only the last sample from each cow was used.

\begin{tabular}{llccc}
\hline Sample round & Study period & Cows sampled & Samples used & Frequency \\
\hline 1 & Aug 1999 to Jan 2000 & 6620 & 2975 & $24 \%$ \\
2 & Sept 2000 to Feb 2001 & 6410 & 3000 & $24 \%$ \\
3 & Oct 2001 to March 2002 & 6670 & 6655 & $52 \%$ \\
Total & & 19,700 & 12,630 & $10 \% \%$ \\
\hline
\end{tabular}

Currently, control of paratuberculosis is based on prevention of bacterial transmission, culling of test-positive animals, and vaccination. Vaccination against paratuberculosis will not necessarily prevent multiplication of Map in the animals, but the prevalence of clinical disease will decrease in herds performing vaccination (Wentink et al., 1994). Vaccination is thought to stimulate the CMI, although the long-term effect on the immune response may vary considerably (Muskens et al., 2002).

Lack of valid diagnostic tests for an early detection of infection is the major reason why control of paratuberculosis is difficult. Particularly in the early stages of infection, it is difficult to determine whether infection is present, and to determine whether disease will ever develop to a stage affecting the cow or the performance of the cow. Two main principles for detection are used. One is through detection of an immune response (e.g., antibody ELISA), and the other is detection of the agent (e.g., by cultivation of the bacteria) (Nielsen et al., 2001).

It has been known for years that in both humans and animals there are differences in the susceptibility towards certain diseases. A difference in susceptibility towards tuberculosis in humans caused by Mycobacterium tuberculosis has shown to be under the influence of genes (Abel and Casanova, 2000). Mycobacterium avium subsp. avium (Maa) is closely related to Map, and the natural resistance associated macrophage protein 1 (NRAMP1) gene has been shown to play a role in resistance towards Maa in mice (Vidal et al., 1993).

Some infected cows do not develop paratuberculosis; there could be differences in genetic susceptibility. A Dutch study evaluated the heritability of paratuberculosis among vaccinated and unvaccinated animals (Koets et al., 2000), where the diagnoses were based on postmortem examinations. For vaccinated cows, the estimated heritability of 0.09 was found, whereas a heritability of less than 0.01 was found for unvaccinated cows. Another study estimated the heritability of paratuberculosis in goats, also based on postmortem investigations, and the heritability ranged from 0.01 to 0.15 in different breeds (Singh et al., 1990). As pathologic lesions may appear both in cows with CMI and those with HI (Payne and Rankin, 1961), these findings re- flect heritability of pathology and not the heritability of the ability to mount antibodies. Given that HI occurs prior to or concomitantly with clinical disease, it would be of interest if the immunologic patterns are heritable. However, apparently no studies on the heritability of these more specific immunological aspects of paratuberculosis have been performed.

The aim of this study was to estimate the genetic variation and heritability of the ability to produce IgG against paratuberculosis in dairy cattle.

\section{MATERIALS AND METHODS}

\section{Milk Samples}

The milk samples were collected through the Danish Kongeå project (Andersen et al., 2000). Of approximately 260 herds in the area covered by the project, 110 herds gave permission to test their animals, and of these, 99 herds had Danish Holsteins. None of the herds used vaccination against paratuberculosis. The study area and herds are described in further details elsewhere, including a nonresponse analysis (Nielsen et al., 2002b). The milk samples were taken from all lactating cows in the herds through the Danish recording scheme.

Samples from the herds were collected in 3 crosssectional samplings with approximately 13 mo between each sampling in the period August 1999 to April 2002 (Table 1). In the first sampling, 99 herds participated, while only 95 and 91 herds participated in the following 2 samplings. Two of the initial herds withdrew from the project after the first sampling, and 4 herds ceased production each year.

Because of high replacement rates for animals in dairy herds, the number of animals would decrease dramatically if followed over a period of time. However, older cows are more likely to have developed antibodies against Map than younger cows. Therefore, we used only the sample from the last period in which a specific cow was still present in the herd. The last samples were selected to allow as many cows as possible to develop antibodies. A total of 19,700 milk samples were collected, among which 12,630 samples were last samples (Table 1). 
Table 2. Data editing criteria and the resulting number of samples for analysis.

\begin{tabular}{lclc}
\hline Reason for reduction & Reduction & No. of animals left & Percent \\
\hline All cows & - & 12,630 & 100.0 \\
Age at first calving > $1400 \mathrm{~d}$ & 16 & 12,614 & 99.9 \\
DIM < 6 d and DIM > 400 d & 921 & 11,693 & 92.6 \\
Missing date of birth, unknown pedigree ${ }^{1}$ & 146 & 11,547 & 91.4 \\
No recorded milk production & 1 & 11,546 & 91.4 \\
Missing date of first calvings & 9 & 11,535 & 91.4 \\
Resulting no. of samples & 1065 & 11,535 & 91.4 \\
\hline
\end{tabular}

${ }^{1}$ Missing date of birth and missing information of both sire and dam.

The samples were treated and analyzed with a milk antibody ELISA for detection of IgG as described in a previous paper (Nielsen, 2002). ELISA measures the amount of antibodies against Map and possibly certain other mycobacteria as an optical density (OD). The ELISA is not specific for Map IgG, but cross-reactivity toward other mycobacteria was reduced by absorption by addition of Mycobacterium phlei (a closely related mycobacterium) to the milk samples though this method does not assure 100\% specificity. Each ELISA plate was numbered to allow inclusion of the laboratory variation (Nielsen, 2002).

Information on parity, age at first calving, DIM, and milk production was retrieved from the Danish Cattle Database. Because the OD had a skewed frequency distribution, a log transformation was performed and named $\ln (\mathrm{OD})$.

\section{Data Editing}

Parity and DIM. Records excluded for various reasons are listed in Table 2. Samples from cows with DIM $<6 \mathrm{~d}$ when the sample was taken were excluded as colostrum had earlier been found unsuitable for ELISA testing (unpublished data). Also, samples from cows with DIM $>400$ were also excluded. Reasons were that long DIM had earlier shown abnormal OD (unpublished data) and an excessive DIM might be due to a failure in calving reports.

Parity, DIM, and age at first calving reflect the age of the cow, and since paratuberculosis has a very long incubation period, younger cows are not as likely to have detectable antibodies. Therefore the cows were divided into 3 parity groups: parity $=1$, parity $=2$, and parity $>2$. The ranges of parities were from 1 to 13 , with $37 \%$ of the cows from parity $1,27 \%$ of the cows from parity 2 , and $36 \%$ from third and higher parities. The likelihood of being positive in higher parities is 2 to 4 times the likelihood of testing positive in first parity (Nielsen et al., 2002a) and increases with increasing DIM in infected animals (Nielsen et al., 2002c). Sum- mary statistics of selected variables are given in Table 3 .

Groups of dam-daughter pairs and maternal sisters. The milk samples tested comprised both mother cows with one or several daughters as well as groups of maternal sisters whose mothers did not have a measurement of paratuberculosis present in the data. To evaluate a possible effect on the antibody-level from dams with intrauterine infections, 2 alternative classifications were considered. Dams and their daughters were grouped, resulting in 7617 groups. Alternatively, maternal sibs were grouped, resulting in 9423 groups.

Pedigree information. Pedigree information on the 11,535 animals left after data editing was obtained from the Danish Cattle Database, and pedigrees were traced as far back as possible. This resulted in a pedigree data set comprising 40,341 animals with information on sire, dam, and date of birth.

\section{Statistical Analyses}

Test and selection of the fixed effects was done using Proc Mixed in SAS v.8 (SAS, 1999), while the random effects were tested and selected using the program package DMU (Madsen and Jensen, 2002). After the variables were selected, the final analyses were based on a bivariate model with OD and daily milk yield as dependent variables. In addition a univariate animal model using the complete pedigree was compared with a univariate sire model ignoring genetic relationships other than through the sire.

The genetic analysis was made by DMU (Jensen et al., 1997; Madsen and Jensen, 2002) using an average information restricted maximum likelihood (AI-REML) algorithm for estimation of (co)variance components in mixed models (Jensen et al., 1997).

The fixed effects considered were parity, DIM, age at first calving, herd, and sample round. These were all significant in the F-test (at 5\% level). Interactions between the fixed effects were also tested. Selection of the random effects was based on likelihood ratio tests of nested models. Random effects considered were ELISA 
Table 3. Minimum, maximum, mean, median, and standard deviation (SD) of the logarithmic transformation of optical density (OD), daily milk yield, and age at first calving for the 11,535 animals that were left after data editing.

\begin{tabular}{lccccc}
\hline & Minimum & Maximum & Mean & Median & SD \\
\hline Ln(OD) & -5.17 & 0.92 & -2.32 & -2.43 & 0.89 \\
Kg Milk & 2.2 & 56.6 & 24.7 & 24.3 & 7.7 \\
Age at first calving & 495 & 1399 & 860 & 842 & 101 \\
\hline
\end{tabular}

plate in addition to additive genetic effects and maternal groups.

It is expected that the antibody level in milk is influenced by daily milk yield. To account for this, a bivariate model was fitted, with daily milk yield $(\mathrm{kg})$ and antibody level $(\ln (\mathrm{OD}))$ as response variables. The model in matrix notation was:

$$
\left(\begin{array}{l}
Y_{1} \\
Y_{2}
\end{array}\right)=X \beta+Z a+H p+W c+e
$$

where $Y_{1}$ is a vector of log transformed $\mathrm{OD}, Y_{2}$ is a vector of observed milk yield at the day the milk sample was taken. $\mathrm{X}$ is a design matrix relating fixed effects to observations, and $\beta$ is a vector of fixed effects for each trait including fixed effects of parity nested within sampling round, herd nested within sampling round, a linear regression on age at first calving and a curvilinear regression on DIM at the day of sampling. $\mathrm{Z}$ is a design matrix relating random additive genetic effects $a$ to observations. $\mathrm{H}$ is a design matrix relating ELISA plate number effects $p$ to observations and $\mathrm{W}$ is a design matrix relating random groups effects $c$ (dam-daughter or maternal sibs) to observations, and e is the residual. Random effects was assumed independent with zero expectations and (co)variance structures as

$$
\begin{aligned}
& \operatorname{Var}(a)=G \otimes A \\
& \operatorname{Var}(p)=P \otimes I \\
& \operatorname{Var}(c)=C \otimes I \\
& \operatorname{Var}(e)=R \otimes I
\end{aligned}
$$

where $\mathrm{A}$ is the numerator relationship matrix, I are identity matrices of proper order and G, P, C, and R are $2 \times 2$ covariance matrices for additive genetic, ELISA plate number, group and residual effects respectively. $\otimes$ is the direct product operator. Group effects were nonsignificant; the final model did not include group effects.

Sire model. A sire model was also fitted to estimate the heritability by using only the pedigree of sires of the cows rather than the entire pedigree. This was done by SAS using mixed models. The univariate animal model from the DMU analyses with only $\ln (\mathrm{OD})$ as response variable was used for comparison with the results from the sire model.

\section{RESULTS}

The 2 groups, dam-daughters and maternal sisters, were tested as random effects and both came out as nonsignificant. Common sources of variation between dam-daughter pairs or maternal sister groups explained less than $1 \%$ of the total variation. The random effect of the ELISA plate showed a variance of 0.139 $(\mathrm{SD}=0.013)($ Table 4$)$, accounting for $21 \%$ of the total variation in $\ln (\mathrm{OD})$, indicating that laboratory variation have influenced the results as reported earlier (Nielsen, 2002).

The heritability based on the multivariate model was 0.102 , based on the information from Table 4 . For reasons of comparability with other studies phenotypic variance was calculated as the sum of genetic and residual variation. The variation due to ELISA plate number was not considered, as this was a variation due to the specific setup of the laboratory test used in this study. The statistical analyses showed a nonsignificant genetic correlation between daily milk yield and antibody level $(\ln (\mathrm{OD}))$ of -0.037 with a SD of 0.13 (Table 4).

The univariate model using the whole pedigree showed a significant heritability of 0.101 . In the sire model, the sire variance was 0.01187 , with $\mathrm{SD}=0.003$. When calculating the heritability of the sire-model, the estimated heritability was 0.091 . The difference between using the complete pedigree or using only the sire as the pedigree was small.

\section{DISCUSSION}

If paratuberculosis is heritable, genetic manipulation may become an element in disease control. In the present study, a heritability of humoral antibody response of 0.10 was found, indicating that the genes in some way influence the chance of returning to the undesired humoral immune response where the immune system apparently does not control the infection any longer. Other factors such as stress, however, may also influence the development of disease. The calculated heritability, based on ELISA test results, provided informa- 
Table 4. Variance components for Mycobacterium avium subsp. paratuberculosis antibody level $(\ln (\mathrm{OD}))$ and daily milk yield (kg milk). ${ }^{1}$

\begin{tabular}{llll}
\hline Variance component & $\operatorname{Ln}(\mathrm{OD})$ & Kg milk & Correlation \\
\hline ELISA plate-number & $0.139(\mathrm{SD}=0.013)$ & - & - \\
Genotype & $0.054(\mathrm{SD}=0.011)$ & $3.54(\mathrm{SD}=0.64)$ & $-0.037(\mathrm{SD}=0.13)$ \\
Residual & $0.473(\mathrm{SD}=0.011)$ & $27.0(\mathrm{SD}=0.61)$ & $-0.178(\mathrm{SD}=0.02)$ \\
\hline
\end{tabular}

${ }^{1} \mathrm{OD}=$ Optical density, $\mathrm{SD}=$ asymptotic standard deviation.

tion about the genetic capability of producing an immune response. The heritability estimate does not reveal information on the genetic variation in clinical symptoms, but it may be an approximation of the ability to control the infection.

The low and nonsignificant genetic correlation $(-0.037)$ between antibody level and daily milk yield indicates that the genes influencing the paratuberculosis antibody production do not influence the milk yield. Thus, it should be possible to breed towards better milk yield and against paratuberculosis at the same time.

There has been evidence that intrauterine infection of fetuses is possible (Seitz et al., 1989; Sweeney et al., 1992). This was one reason for including the variable groups, consisting of milking dam-daughters and milking maternal sisters, in the analyses. If antibodies to Map were seen more often in groups of maternal sisters and dam-daughters, it might partly be explained hereby. In this study, neither of the 2 groups came out as significant factors. In the present study, the nonsignificant effect of dam-daughter pairs and maternal sib groups could have been affected by the age-structure of the population. However, the samples used were taken with a majority of cows from second and higher parities, where end-stage disease could easily have occurred. Hence this effect is not very likely. A reasonable explanation for the effect of zero of the maternal sister groups and dam may be that only cows with clinical or endstage disease will regularly infect the offspring as concluded by Sweeney et al. (1992).

As described earlier, only a few other studies have described the genetic variation of paratuberculosis in cattle. Whether genetic selection is used in a program of controlling a certain disease, depends both on the size of the heritability and on the importance and interest of the disease. Mastitis for example has a relatively low heritability, but because of the importance and economical loss due to this disease, mastitis is included in the selection index in some countries.

The main difference between the present and other studies on heritability of paratuberculosis is the way the diagnoses or the detection of paratuberculosis was done. In both the study by Koets et al. (2001) and the study on goats (Singh et al., 1990), postmortem examination was used for detection of paratuberculosis. This is probably an overall more valid method for detection of infection, but it does not include different stages of the infection to the same degree as does detection of antibody level, thus making the interpretation of the biological aspects behind it more obscure. Most of the animals used by Koets et al. (2001) were vaccinated. It is known that the vaccinated cows still may shed Map, and, therefore, it would be possible that some of the pathological changes in the vaccinated animals do not lower the performance of the animal, although this has to our knowledge not been studied.

In the present study, it was chosen to use the last sample from each cow because of a higher likelihood of antibody response in late samples. To test whether this selection had influenced the heritability found, another estimation was done on the OD, where the sample was selected randomly from each cow. This yielded a heritability of 0.096 (data not shown) instead of 0.101 , where both the residual (0.45) and the genetic variation (0.048) decreased. In consequence, the selection had had no noteworthy influence on the heritability found.

Results of other studies show that experimentally infected animals show the highest heritability of disease susceptibility. When using experimental infection in a study, it is possible to control exposures to pathogens, and, at the same time, the environmental aspects can be kept constant. A paratuberculosis infection requires exposure to a certain amount of Map. It is assumed that animals with an ELISA response have had some level of exposure to Map. However, Map antibody ELISA are known to be less than $100 \%$ specific. A reasonable specificity estimate for the ELISA used in the present study is 96 to $98 \%$, although in general it appears to be more specific when used for first-parity cows compared with older cows (Nielsen and Toft, 2002). The sensitivity of the ELISA is affected by stage of disease and may vary from 20 to $80 \%$. However, using the ELISA response on a continuous scale decreases the importance hereof.

There are large differences in prevalences among the herds in this study, and it is unlikely that all animals had been exposed to Map. The environmental circumstances will not have been the same for all animals. In experimental infections, the heritability might therefore have been found higher. 
In conclusion, this study demonstrated a significant heritability of antibody production to paratuberculosis (0.10), with no significant genetic correlation between daily milk yield and level of antibodies against paratuberculosis. The OD did not allow demonstration of genetic variation in antibody response for dam-daughter pairs and maternal sisters, as might have been expected because of possible intrauterine infection.

\section{ACKNOWLEDGMENTS}

The Kongeå-project of the Danish Cattle Federation has financed the milk sampling and the laboratory examinations in this study.

\section{REFERENCES}

Abel, L., and L. J. Casanova. 2000. Genetic predisposition to clinical tuberculosis: Bridging the gap between simple and complex inheritance. Am. J. Hum. Genet. 67:274-277.

Andersen, H. J., K. Aagaard, F. Skjøth, E. Rattenborg, and C. Enevoldsen. 2000. Integration of research, development, health promotion, and milk quality assurance in the Danish dairy industry. Pages 258-260 in Proc. Ninth Symp. Int. Soc. Vet. Epidemiol. Econ. M. D. Salman, P. Morley, and R. Ruch-Gallie, eds. Breckenridge, $\mathrm{CO}$.

Chi, J., J. A. VanLeeuwen, A. Wentink, and G. P. Keefe. 2002. Direct production losses and treatment costs from bovine viral diarrhoea virus, bovine leukosis virus, Mycobacterium avium subspecies paratuberculosis and Neospora caninum. Prev. Vet. Med. 55:137-153.

Chiodini, R. J. 1996. Immunology: Resistance to paratuberculosis. Vet. Clin. North Am. Food. Anim. Pract. 12:313-343.

Jensen, J., E. A. Mantysaari, P. Madsen, and R. Thomson. 1997. Residual maximum likelihood estimation of (co)variance components in multivariate mixed linear models using average information. J. Indian Soc. Agric. Stat. 49:215-236.

Kennedy, D. J., and G. Benedictus. 2001. Control of Mycobacterium avium subsp. paratuberculosis infection in agricultural species. Rev. Sci. Tech. 20:151-179.

Koets, A. P., G. Adugna, L. L. G. Janss, H. J. van Weering, C. H. J. Kalis, G. H. Wentink, V. P. M. G. Rutten, and Y. H. Schukken. 2000. Genetic variation of susceptibility to Mycobacterium avium subsp. paratuberculosis infection in dairy cattle. J. Dairy Sci. 83:2702-2708.

Koets, A. P., V. P. M. G., Rutten, M. de Boer, D. Bakker, P. ValentinWeigand, and W. van Eden. 2001. Differential changes in heat shock protein-, lipoarabinomannan-, and purified protein derivative-specific immunoglobulin G1 and G2 isotype responses during bovine Mycobacterium avium subsp. paratuberculosis infection. Infect. Immun. 69:1492-1498.
Madsen, P., and J. Jensen. 2002. A user's guide to DMU. A package for analysing multivariate mixed models. Version 6 . release 4.4 Danish Institute of Agricultural Sciences, Tjele, Denmark.

Muskens, J., F. van Zijderveld, A. Eger, and D. Bakker. 2002. Evaluation of the long-term immune response in cattle after vaccination against paratuberculosis in two Dutch dairy herds. Vet. Microbiol. 86:269-278.

Nielsen, S. S., K. K. Nielsen, A. Huda, R. Condron, and M. T. Collins. 2001. Diagnostic techniques for paratuberculosis. Bull. IDF 362:5-17.

Nielsen, S. S. 2002. Variance components of an enzyme-linked immunosorbent assay for detection of IgG antibodies in milk samples to Mycobacterium avium subspecies paratuberculosis in dairy cattle. J. Vet. Med. B 49:384-387.

Nielsen, S. S., and N. Toft. 2002. Optimisation of the validity of ELISA and faecal culture tests for paratuberculosis: Selection of population or correction by population characteristics? Pages 400-405 in R. A. Juste, M. V. Geijo, and J. M. Garrido, eds. Proc. Seventh Int. Colloquium on Paratuberculosis, Bilbao, Spain.

Nielsen, S. S., C. Enevoldsen, and Y. T. Gröhn. 2002a. The Mycobacterium avium subsp. paratuberculosis ELISA response by parity and stage of lactation. Prev. Vet. Med. 54:1-10.

Nielsen, S. S., Y. T. Gröhn, R. L. Quaas, and J. F. Agger. 2002b. Paratuberculosis in dairy cattle: Variation of the antibody response in offspring attributable to the dam. J. Dairy Sci. $85: 406-412$.

Nielsen, S. S., Y. T. Gröhn, and C. Enevoldsen. 2002c. Variation of the milk antibody response to paratuberculosis in naturally infected dairy cows. J. Dairy Sci. 85:2795-2802.

Ott, S. L., S. J. Wells, and B. A. Wagner. 1999. Herd-level economic losses associated with Johne's disease on US diary operations. Prev. Vet. Med. 40:179-192.

Payne, J. M., and J. D. Rankin. 1961. The pathogenesis of experimental Johne's disease in calves. Res. Vet. Sci. 2:167-174.

SAS Institute, Inc. 1999. Cary, NC.

Seitz, S. E., L. E. Heider, W. D. Hueston, S. Bech-Nielsen, D. M. Rings, and L. Spangler. 1989. Bovine fetal infection with Mycobacterium paratuberculosis. J. Am. Vet. Med. Assoc. 15:1423-1426.

Singh, N., S. N. Kala, V. S. Vihan, and V. S. Singh. 1990. Genetic study on susceptibility to Johne's disease in goats. Indian J. Anim. Sci. 60:1163-1165.

Stabel, J. R. 2000. Transitions in immune responses to Mycobacterium paratuberculosis. Vet. Microbiol. 77:465-73.

Sweeney, R. W., R. H. Whitlock, and A. E. Rosenberger. 1992. Mycobacterium paratuberculosis isolated from fetuses of infected cows not manifesting signs of the disease. Am. J. Vet. Res. 53:477-480.

Taylor, A. W. 1953. Experimental Johne's disease in cattle. J. Comp. Pathol. 63:355-367.

Vidal, S. M., D. Malo, K. Vogan, E. Skamene, and P. Gros. 1993. Natural resistance to infection with intracellular parasites: Isolation of a candidate for Bcg. Cell 7:469-485.

Wentink, G. H., J. H. Bongers, A. A. P. A. Zeeuwen, and A. Jaartsveld. 1994. Incidence of paratuberculosis after vaccination against $M$. paratuberculosis in two infected dairy herds. J. Vet. Med. B. 41:517-522.

Whitlock, R. H., and C. Buergelt. 1996. Preclinical and clinical manifestations of paratuberculosis (including pathology). Vet. Clin. North Am. Food Anim. Pract. 12:345-356. 